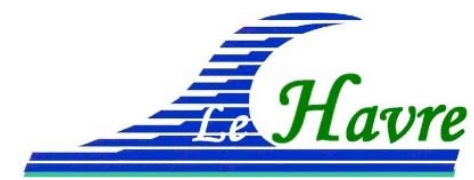

XVI èmes Journées Nationales Génie Côtier - Génie Civil

Le Havre, 2020

DOI:10.5150/jngcgc.2020.010 C Editions Paralia CFL

disponible en ligne - http://www.paralia.fr - available online

\title{
Effet de la végétation sur la dissipation des vagues au niveau d'un pré salé
}

\section{Laura LAVAUD ${ }^{1}$, Antoine LECHEVALIER ${ }^{2}$, Thibault COULOMBIER ${ }^{\text {, }}$ Xavier BERTIN ${ }^{1}$, Kévin MARTINS ${ }^{3,1}$}

\author{
1. UMR 7266 LIENSs, CNRS/La Rochelle Université, 2 rue Olympe de Gouges, \\ 17000 La Rochelle, France. \\ laura.lavaud@univ-lr.fr
}

2. Unité Territoriale de St-Barthélémy et St-Martin de la DEAL Guadeloupe, 23 rue de Spring - Marigot, 97150 Saint-Martin, Guadeloupe, France.

3. UMR 5805 EPOC, CNRS/Université de Bordeaux, Allée Geoffroy Saint-Hilaire, 33615 Pessac, France.

\section{Résumé :}

Cette étude analyse l'effet de la végétation sur l'atténuation des vagues en combinant mesures in-situ et modélisation numérique. Une campagne de mesures a été réalisée sur un pré salé situé dans la Baie de Marennes-Oléron (Côte Atlantique française) en conditions de vagues modérées, caractérisées par des hauteurs significatives et des périodes de pic atteignant $0.65 \mathrm{~m}$ et $4 \mathrm{~s}$, respectivement. La zone étudiée comporte deux espèces de plantes principales : la spartine (Spartina maritima) et l'obione (Halimione portulacoides). L'analyse des données révèle une atténuation de la hauteur des vagues de $1.3 \% / \mathrm{m}$, valeur semblable à celles reportées dans la littérature. Un système de modélisation numérique couplé vagues-courant est implémenté à l'échelle des Pertuis Charentais, afin de reproduire les conditions incidentes sur le pré-salé puis d'évaluer l'effet de la végétation sur la propagation des vagues représenté par la formulation de MENDEZ \& LOSADA (2004). Les résultats montrent que la prise en compte de la dissipation des vagues par la végétation dans le modèle améliore de $85 \%$ la prédiction de l'évolution de la hauteur des vagues sur le transect instrumenté. Ces résultats mettent en avant le rôle important de la végétation halophile dans l'atténuation de l'énergie des vagues et son potentiel de protection du littoral lors des évènements extrêmes.

\section{Mots-clés :}

Végétation, Dissipation des vagues, SCHISM, Protection côtière.

\section{Introduction}

Les effets combinés de l'élévation du niveau de la mer associée au changement climatique et de la croissance démographique dans les zones littorales augmentent les risques de submersions marines. Face à ce constat, les systèmes de protection des côtes doivent s'adapter, en intégrant notamment de nouvelles stratégies de défense. Dans les zones littorales, la végétation halophile peuplant les marais salés et les zones intertidales, ou 


\section{Thème 1 - Hydrodynamique côtière}

encore les forêts de mangroves, atténuent l'énergie des vagues (DALRYMPLE et al., 1984 ; MENDEZ \& LOSADA, 2004 ; MÖLLER, 2006) et peuvent limiter les surcotes (TEMMERMAN et al., 2013). Ces processus suscitent un intérêt croissant dans la communauté scientifique internationale et l'utilisation potentielle de ces écosystèmes comme solution "douce" de protection du littoral est de plus en plus considérée (SUTTON-GRIER et al., 2015). La dissipation des vagues par la végétation dépend du climat de vagues, des caractéristiques des plantes, mais aussi de l'hydrodynamisme local, qui contrôle le type et la répartition de celle-ci. Malgré la multiplicité de facteurs qui rend complexe la compréhension des interactions entre l'hydrodynamique littorale et la végétation, de nombreuses études ont contribué à améliorer ces connaissances grâce au déveloement de modèles numériques (MENDEZ \& LOSADA, 2004 ; SUZUKI et al., 2012 ; VAN ROOIJEN et al., 2015) et à des expérimentations (DUBI \& TORUM, 1995 ; MÖLLER, 2006). Cependant, la plupart de ces expérimentations se basent sur des mesures effectuées en laboratoire avec une végétation synthétique, ce qui peut poser le problème de la représentativité des résultats dans le milieu in-situ, particulièrement lors d'évènements extrêmes.

L'objectif de cette étude est donc de quantifier l'effet de la végétation sur la dissipation des vagues en conditions in-situ. Pour ce faire, une campagne de mesures a été réalisée sur un pré salé se déveloant dans la Baie de Marennes-Oléron (Côte Atlantique française) dans des conditions de vagues modérées. L'analyse des données est complétée par des simulations numériques réalisées avec un système de modélisation couplant un modèle spectral de vagues et un modèle de circulation.

\section{Méthodes}

\subsection{Zone d'étude}

La zone d'étude se situe sur la Côte Atlantique française, dans le département de la Charente-Maritime (figure 1). Elle est localisée dans la Baie de Marennes-Oléron, sur l'estran de Brouage. La zone intertidale de la vasière de Brouage est large de $4 \mathrm{~km}$ $\left(42 \mathrm{~km}^{2}\right)$ avec une pente moyenne de $\sim 1 / 800$ et des courants de marée transversaux de $\sim 0.3 \mathrm{~m} . \mathrm{s}^{-1}$ à $\sim 0.5 \mathrm{~m} . \mathrm{s}^{-1}$ (LE HIR et al., 2000). En amont de la vasière, un pré salé se déveloe le long du rivage et se compose du bas vers le haut : de spartine maritime, de puccinellie maritime, d'obione et de soude arbustive. Le régime tidal est semi-diurne, macro-tidal, avec un marnage compris entre $1.10 \mathrm{~m}$ et $6.60 \mathrm{~m}$. Bien que la vasière de Brouage soit protégée de la houle par l'Ile d'Oléron, un fetch de plusieurs dizaines de kilomètres peut se déveloer dans la zone sous l'effet de vents de Nord-Ouest et ainsi générer des vagues pouvant atteindre $1 \mathrm{~m}$ (LE HIR et al., 2000). 


\section{XVİ̀mes Journées Nationales Génie Côtier - Génie Civil \\ Le Havre 2020}
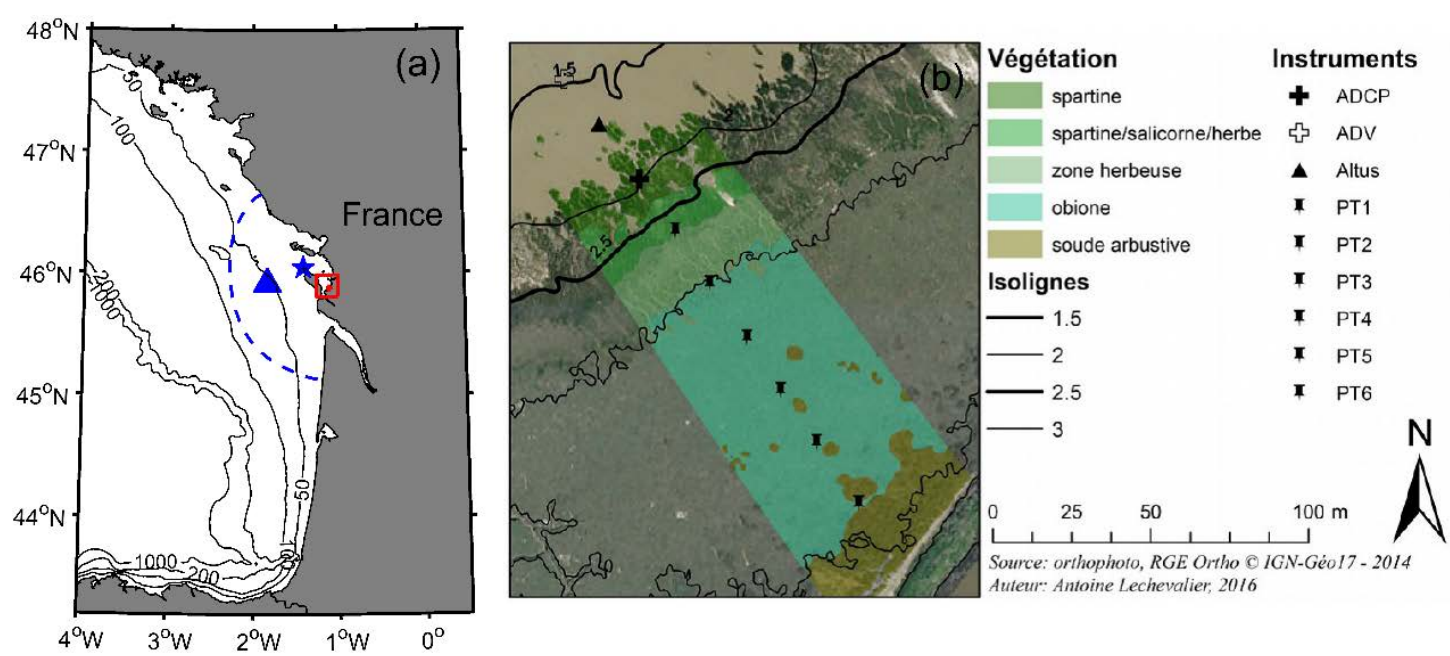

Figure 1. Localisation de la zone d'étude (rouge), de la bouée Oléron Large (triangle bleu), de la station météorologique (étoile bleue) et de la frontière ouverte de la grille de calcul (bleu) (a). Position des capteurs et types de végétation sur la zone d'étude (b).

\subsection{Campagne de mesures et caractérisation de la végétation}

La campagne de mesures a été réalisée du 8 au 11 mars 2016 en période de vive-eau, sur un transect de $160 \mathrm{~m}$ de long perpendiculaire à la bathymétrie (figure 1), avec une pente moyenne de $0.8 \%$. Un courantomètre ADV, un altimètre Altus, un profileur de courant ADCP ainsi que six capteurs de pression (PT) mesurant à $4 \mathrm{~Hz}$, ont été déployés avec un espacement d'environ $20 \mathrm{~m}$. Les PT ont été placés à la surface de l'estran, dans des tubes en inox enfoncés de $0.80 \mathrm{~m}$. L'ADCP et l'ADV, également équipés de capteurs de pression mesurant à 2 et $16 \mathrm{~Hz}$ respectivement, ont été disposés dans des structures en inox vissées dans l'estran. La position des instruments ainsi que la topographie ont été relevées au GNSS différentiel. Des vents forts de Nord-Ouest ont été enregistrés dans les Pertuis Charentais pendant la campagne avec une vitesse maximale de $17 \mathrm{~m} \cdot \mathrm{s}^{-1}$ (rafales à $24 \mathrm{~m} . \mathrm{s}^{-1}$ ) le 9 mars, permettant de déveloer des vagues de l'ordre de $0.65 \mathrm{~m}$ sur la zone d'étude. Afin de représenter la dissipation des vagues par la végétation dans le modèle, il est nécessaire d'en définir les caractéristiques (tableau 1). Plusieurs mesures ont été effectuées sur la zone d'étude, puis moyennées.

Tableau 1. Caractéristiques des principaux végétaux présents sur la zone d'étude.

\begin{tabular}{lcc}
\hline & Spartine & Obione \\
\hline Hauteur $(m)$ & $0.27 \pm 0.05$ & $0.80 \pm 0.20$ \\
Densité (plants. $m^{-2}$ ) & $990 \pm 10$ & $680 \pm 10$ \\
Aire d'un plant par unité de hauteur $(m)$ & $0.032 \pm 0.002$ & $0.03 \pm 0.01$ \\
\hline
\end{tabular}




\section{Thème 1 - Hydrodynamique côtière}

\subsection{Analyse des données}

Les signaux de pression au fond issus des différents capteurs ont d'abord été corrigés des variations de la pression atmosphérique mesurée à la station météorologique de Chassiron (figure 1). Chaque signal est organisé en échantillons de $15 \mathrm{~min}$ pour les PT et l'ADV, et en échantillons de $8 \mathrm{~min} 30 \mathrm{~s}$ pour l'ADCP. Une analyse spectrale par transformée de Fourier discrète est ensuite aliquée aux échantillons afin de calculer les spectres de densité d'énergie $\left(E_{p}(f)\right)$. Un fenêtrage de Hann est utilisé ainsi qu'une aroche par sous-blocs : 10 sous-blocs sont considérés pour les PT et l'ADV et 5 pour l'ADCP (soit 20 et 10 degrés de liberté respectivement). Les spectres de densité d'énergie sont ensuite corrigés de l'atténuation de la pression avec la profondeur d'après la théorie linéaire pour obtenir des spectres d'élévation de la surface libre $(E(f))$. La hauteur significative spectrale $\left(H_{m 0}\right)$ et la période moyenne des vagues ( $\left.T_{m 02}\right)$ sont alors calculées comme suit :

$$
H_{m 0}=4 \sqrt{m_{0}} \quad \text { (1) } \quad T_{m 02}=\sqrt{\frac{m_{0}}{m_{2}}}
$$

Où $m_{n}$ sont les moments d'ordre $n$ :

$$
m_{n}=\int_{f \min }^{f \max } f^{n} E(f) d f
$$

Dans l'équation (3), $f_{\min }$, fixée à $0.04 \mathrm{~Hz}$, correspond à la fréquence de coupure entre les bandes infra-gravitaire et gravitaire. $f_{\max }$, fixée à $0.5 \mathrm{~Hz}$, est la fréquence de coupure haute, définie comme la fréquence maximale au-delà de laquelle l'atténuation du signal avec la profondeur ne peut plus être corrigée.

\subsection{Système de modélisation}

\subsubsection{Description du système de modélisation}

Dans cette étude, les simulations numériques sont réalisées avec le système de modélisation SCHISM (ZHANG et al., 2016). Il réalise le couplage complet entre un modèle de circulation hydrodynamique 3D et le modèle spectral de vagues WWMII (ROLAND et al., 2012) qui résout l'équation de conservation de l'action des vagues. Les deux modèles partagent la même grille non structurée et décomposition du domaine pour la parallélisation. Le modèle de circulation est basé sur l'utilisation de schémas semiimplicites et d'une méthode Eulérienne-Lagrangienne pour résoudre l'advection, ce qui permet de s'affranchir des contraintes de stabilité numérique.

Dans cette étude, le modèle de circulation est utilisé en mode 2DH barotrope et le pas de temps est fixé à $60 \mathrm{~s}$. Il est couplé à WWMII, avec un pas de temps de $600 \mathrm{~s}$, utilisé dans cette étude avec une formulation implicite. Le déferlement bathymétrique est calculé d'après le modèle de BATTJES \& JANSSEN (1978) pour lequel les paramètres $\gamma$ et $\alpha$ ont été fixés à 0.55 et 0.035 respectivement. La valeur de 0.035 pour $\alpha$ correspond à 40 fois $\beta$, la pente de la vasière de Brouage. PEZERAT et al. (2020) montrent qu'au niveau des 


\section{XVIèmes Journées Nationales Génie Côtier - Génie Civil \\ Le Havre 2020}

fonds en pente faible, cette paramétrisation adaptative améliore la prédiction des vagues par raort à une valeur de $\alpha$ par défaut (proche de 1). La grille de calcul couvre la zone des Pertuis Charentais, avec une résolution de $1300 \mathrm{~m}$ à la frontière océanique jusqu'à $5 \mathrm{~m}$ dans la zone d'étude (figure 1). Dans le modèle de circulation, la marée est forcée à la frontière océanique par le modèle de marées globale FES 2014 (LYARD et al., 2017). L'ensemble du domaine est forcé par les champs de vent à $10 \mathrm{~m}$ et de pression atmosphérique au niveau de la mer issus du système de réanalyse CFSR (SAHA et al., 2014). WWMII est aussi forcé par les champs de vent CFSR et par des séries temporelles de spectres directionnels à la frontière océanique, calculées dans une alication régionale du modèle spectral WaveWatchIII.

\subsubsection{Implémentation de la dissipation des vagues par la végétation}

Les vagues se propageant dans une zone végétalisée subissent une perte d'énergie due au travail qu'elles exercent sur les végétaux. Afin de tenir compte de la dissipation des vagues par la végétation dans le système de modélisation, le modèle de MENDEZ \& LOSADA (2004) est adapté au modèle spectral de vagues WWMII comme suit (voir aussi SUZUKI et al., 2012) :

Avec

$$
S_{d s, v e g}(\sigma, \theta)=\frac{D_{t o t}}{E_{\text {tot }}} E(\sigma, \theta)
$$

$$
D_{t o t}=-\frac{1}{2 g \sqrt{\pi}} \tilde{C}_{d} b_{v} N\left(\frac{g \tilde{k}}{2 \tilde{\sigma}}\right)^{3} \frac{\sinh ^{3} \tilde{k} \alpha h+3 \sinh \tilde{k} \alpha h}{3 \tilde{k} \cosh ^{3} \tilde{k} h} H_{r m s}{ }^{3}
$$

où $H_{r m s}$ est la hauteur quadratique des vagues, $E_{\text {tot }}=H_{r m s}{ }^{2} / 8$ et $E(\sigma, \theta)$ la densité d'énergie totale et discrète des vagues, avec $\sigma$ la fréquence relative et $\theta$ la direction de propagation des vagues. $g$ est l'accélération de la pesanteur, $h$ la hauteur d'eau, $\tilde{C}_{d}$ le coefficient de traînée, $b_{v}$ l'aire d'un plant par unité de hauteur, $N$ la densité des plants, $\alpha . h$ la hauteur des plants, $\tilde{k}$ le nombre d'onde moyen et $\tilde{\sigma}$ la fréquence relative moyenne.

\section{Résultats et discussion}

\subsection{Caractérisation des conditions incidentes à l'ADV}

L'analyse des données expérimentales à l'ADV montre que la hauteur des vagues $H_{m 0}$ incidente (début du transect instrumenté) a atteint $0.65 \mathrm{~m}$ à la marée haute du 9 mars après-midi, pour une période moyenne comprise entre 3 et $4 \mathrm{~s}$ (figure 2). Le modèle reproduit de manière raisonnable les hauteurs d'eau, $H_{m 0}$ et $T_{m 02}$ avec des erreurs normalisées de $10 \%, 16 \%$ et $20 \%$ respectivement (figure 2 ). Ces résultats suggèrent également que le modèle capture correctement l'état de mer bimodal caractérisé par une houle de Nord-Ouest (Période de pic $T p$ de $10 \mathrm{~s}$ et $\mathrm{H}_{m 0}$ atteignant $5 \mathrm{~m}$ mesurés à la bouée Oléron Large, voir figure 1 pour sa localisation) et une mer de vent générée localement par des vents de Nord-Ouest atteignant $17 \mathrm{~m} \cdot \mathrm{s}^{-1}$. 

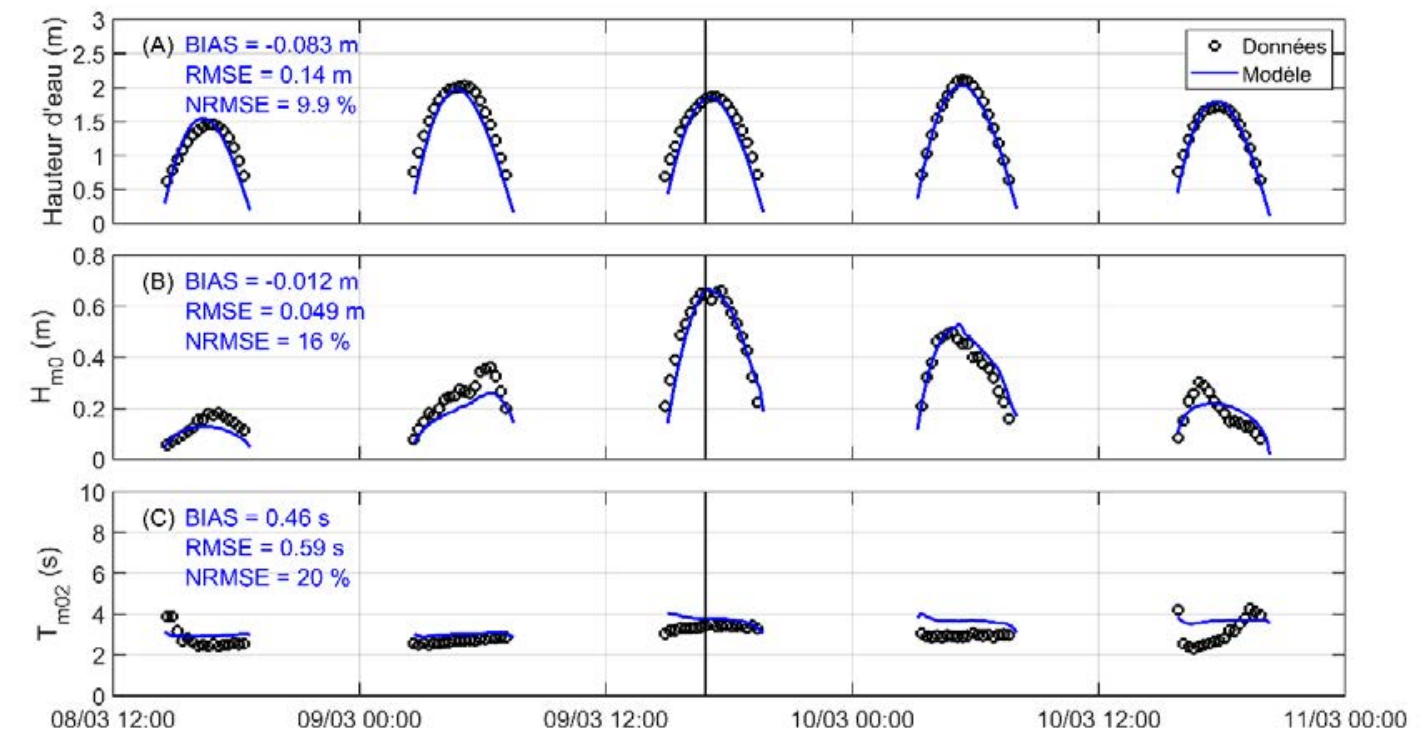

Figure 2. Comparaison de la hauteur d'eau (A), de Hm0 (B) et Tm02 (C) mesurés et modélisés à l'ADV. La ligne verticale définit le pas de temps présenté sur la figure 3.

\subsection{Evolution des vagues sur le transect étudié}

L'analyse des données montre que la hauteur des vagues est réduite de $1.3 \% / \mathrm{m}$ sur les 50 premiers mètres de la zone végétalisée (de $\mathrm{x}=30$ à $80 \mathrm{~m}$ sur la figure 3 ), valeur semblable à celles reportées dans la littérature (MÖLLER, 2006; MÖLLER \& SPENCER, 2002), puis complètement atténuée après $70 \mathrm{~m}(<0.05 \mathrm{~m})$.
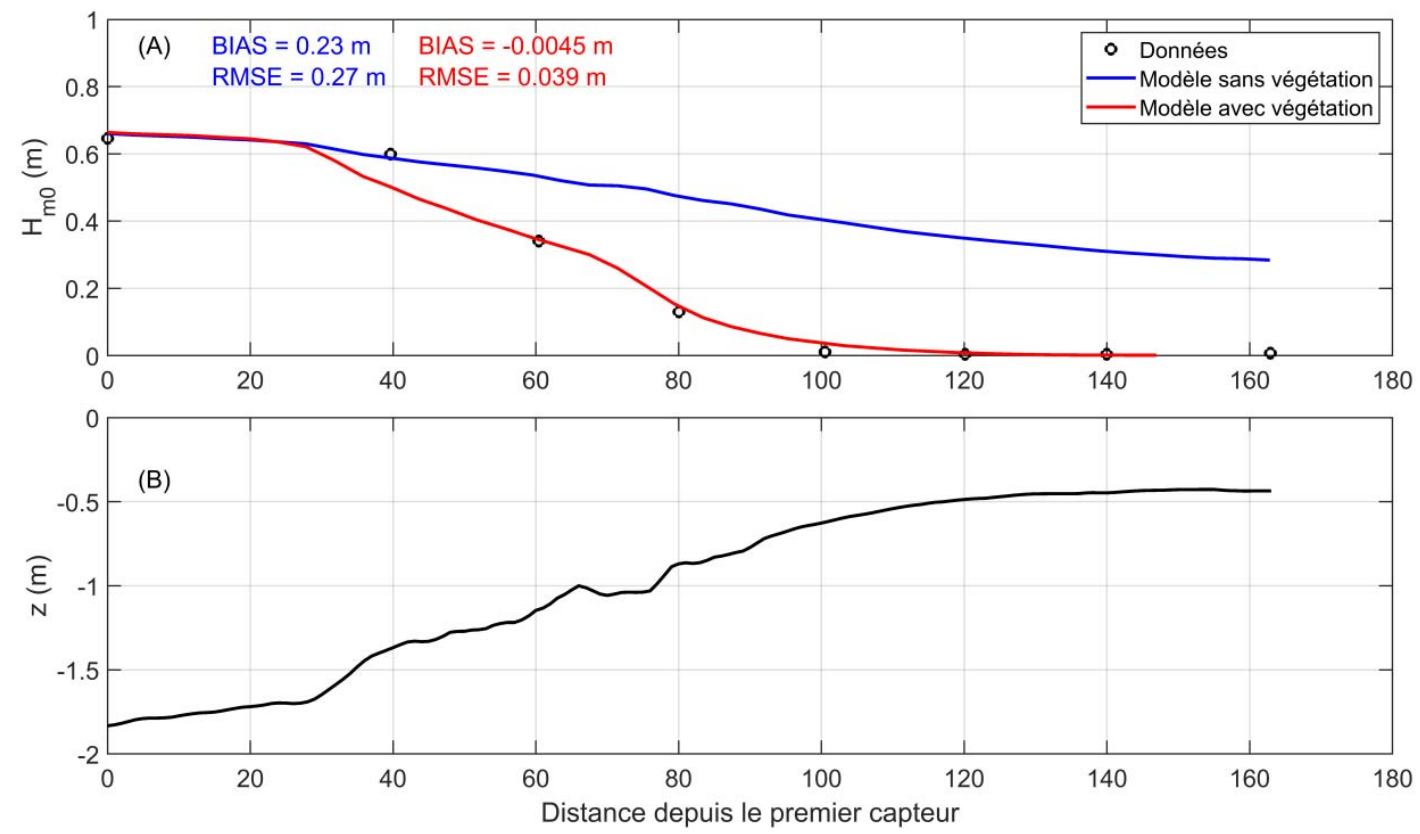

Figure 3. Comparaison de Hm0 mesuré et modélisé, avec (rouge) et sans (bleu) la dissipation des vagues par la végétation, le long du transect à marée haute le 09/03 après-midi (A). Cote du fond par raort à la surface libre (B). 


\section{XVI'̀mes Journées Nationales Génie Côtier - Génie Civil \\ Le Havre 2020}

Afin d'étudier l'effet de la végétation sur la propagation des vagues, deux simulations numériques ont été réalisées en prenant en compte, ou sans le terme de dissipation des vagues par la végétation. Nous considérons dans le modèle que le transect est composé uniquement des deux espèces de plantes principales de l'estran : la spartine (de $x=30$ à 75 $\mathrm{m}$ sur la figure 3 ) et l'obione (de $\mathrm{x}=75 \mathrm{~m}$ jusqu'en haut de l'estran). Selon la formulation de MENDEZ \& LOSADA (2004) pour calculer $C_{d}$, celui-ci est égal à 0.09 pour la spartine et 0.54 pour l'obione. Les résultats révèlent que l'évolution de $H_{m 0}$ est correctement reproduite par le modèle qui tient compte de la végétation avec une erreur quadratique moyenne de $0.04 \mathrm{~m}$ et un biais inférieur à $0.01 \mathrm{~m}$. A l'inverse, le modèle sans végétation fournit une prédiction inexacte de l'évolution de $\mathrm{H}_{\mathrm{m}}$ avec une erreur de l'ordre de 0.30 $\mathrm{m}$ et un bais de $0.20 \mathrm{~m}$ (Figure 3 ). Cette analyse montre que le modèle est capable de reproduire de manière satisfaisante l'atténuation des vagues par la végétation et que celleci peut avoir un rôle prépondérant dans la dissipation des vagues dans les zones littorales propices au déveloement de végétation halophile.

\section{Conclusion et perspectives}

Cette étude a pour objectif d'examiner la dissipation des vagues par la végétation. Le système de modélisation SCHISM-WWMII est aliqué aux mesures réalisées sur un pré salé situé dans la Baie de Marennes-Oléron. La prise en compte de la dissipation des vagues par la végétation dans le modèle améliore de $85 \%$ la prédiction de l'évolution de la hauteur des vagues sur le transect instrumenté par raort à une simulation excluant ce processus. Ce résultat montre alors l'influence importante de la végétation sur la propagation des vagues. Un autre effet de la végétation sur l'hydrodynamique littorale a été identifié par DEAN \& BENDER (2006), qui montrent que la propagation des vagues à travers la végétation peut induire une diminution du niveau moyen, résultant de la propagation de vagues non-linéaires sur un fond très rugueux. L'analyse de nos données expérimentales suggère un phénomène similaire, avec un abaissement du niveau moyen de l'ordre de 0.05-0.10 m. Ces valeurs sont toutefois proches de la marge d'erreur associée à notre protocole expérimental. Cette étude confirme la capacité de la végétation à atténuer l'énergie des vagues et incite à arofondir l'analyse de son action potentielle sur les surcotes. De nouvelles mesures seront réalisées à l'hiver 2020/2021 en conditions plus énergétiques pour vérifier ces résultats.

\section{Références bibliographiques}

BATTJES J. A., JANSSEN J.P.F.M. (1978). Energy loss and set-up due to breaking of random waves. Coastal Engineering Proc., pp 569-587. https://doi.org/10.9753/icce.v16.32

DALRYMPLE R. A., KIRBY J.T., HWANG P.A. (1984). Wave diffraction due to areas of energy dissipation. Journal of Waterway, Port, Coastal, and Ocean Engineering, Vol. 110(1), pp 67-79. https://doi.org10.1061/(ASCE)0733-950X(1984)110:1(67) 


\section{Thème 1 - Hydrodynamique côtière}

DEAN R.G., BENDER C.J. (2006). Static wave setup with emphasis on damping effects by vegetation and bottom friction. Coastal engineering, Vol. 53(2-3), pp 149-156. https://doi.org/10.1016/j.coastaleng.2005.10.005

DUBI A., TØRUM A. (1995). Wave damping by kelp vegetation. In Coastal Engineering 1994, pp 142-156. https://doi.org/10.1061/9780784400890.012

LE HIR P., ROBERTS W., CAZAILlET O., CHRISTIE M., BASSOUllet P., BACHER C. (2000). Characterization of intertidal flat hydrodynamics. Continental shelf research, Vol. 20(12-13), pp 1433-1459. https://doi.org/10.1016/S0278-4343(00)00031-5

LYARD F., CARRERE L., CANCET M., GUILLOT A., PICOT N. (2017) FES2014, a new finite elements tidal model for global ocean, submitted in Ocean Dynamics.

MENDEZ F.J., LOSADA I.J. (2004). An empirical model to estimate the propagation of random breaking and nonbreaking waves over vegetation fields. Coastal Engineering, Vol. 51(2), pp 103-118. https://doi.org/10.1016/j.coastaleng.2003.11.003

MÖLLER I. (2006). Quantifying saltmarsh vegetation and its effect on wave height dissipation: Results from a UK east coast saltmarsh. Estuarine Coastal Shelf Sci., Vol. 69, pp 337-351. https://doi.org/10.1016/j.ecss.2006.05.003

MÖLLER I., SPENCER T. (2002). Wave dissipation over macro-tidal saltmarshes: Effects of marsh edge typology and vegetation change. Journal of Coastal Research, Vol. 36(sp1), pp 506-521. https://doi.org/10.2112/1551-5036-36.sp1.506

PEZERAT M., MARTINS K., BERTIN X. (2020). Modelling storm waves in the nearshore area using spectral models. Journal of Coastal Research, in press.

ROLAND A., ZHANG Y.J., WANG H.V., MENG Y., TENG Y-C., MADERICH V., BROVCHENKO I., DUTOUR-SIKIRIC M., ZANKE U. (2012). A fully coupled 3D wave-current interaction model on unstructured grids. Journal of Geophysical Research: Oceans, Vol. 117(C11). https://doi.org/10.1029/2012JC007952

SAHA S., MOORTHI S., X. WU., J. WANG., S. NADIGA., P.TRIPP, D. BEHRINGER., HOU Y.-T., CHUANG H.-Y., IREDELL M., EK M., MENG J., YANG R.Q., PEÑA MENDEZ M., VAN DEN DOOL H., ZHANG Q., WANG W., CHEN M., BECKER E. (2014). The NCEP climate forecast system version 2. Journal of climate, Vol. 27(6), pp 2185-2208. https://doi.org/10.1175/JCLI-D-12-00823.1

SUTTON-GRIER A.E., WOWK K., BAMFORD H. (2015). Future of our coasts: The potential for natural and hybrid infrastructure to enhance the resilience of our coastal communities, economies and ecosystems. Environmental Science \& Policy, Vol. 51, pp 137-148. https://doi.org/10.1016/j.envsci.2015.04.006

SUZUKI T., ZIJLEMA M., BURGER B., MEIJER M.C., NARAYAN S. (2012). Wave dissipation by vegetation with layer schematization in SWAN. Coastal Engineering, Vol. 59(1), pp 64-71. https://doi.org/10.1016/j.coastaleng.2011.07.006

TEMMERMAN S., DE VRIES M., BOUMA T.J. (2012). Coastal marsh die-off and reduced attenuation of coastal floods: A model analysis. Global and Planetary Change, Vol. 92, pp 267-274. https://doi.org/10.1016/i.gloplacha.2012.06.001 


\section{XVIèmes Journées Nationales Génie Côtier - Génie Civil \\ Le Havre 2020}

VAN ROOIJEN A.A., MCCALL R. T., VAN THIEL DE VRIES J. S. M., VAN DONGEREN A. R., RENIERS A. J. H. M., ROELVINK J. A. (2016). Modeling the effect of wave-vegetation interaction on wave setup. Journal of Geophysical Research: Oceans, Vol. 121(6), pp4341-4359. https://doi.org/10.1002/2015JC011392

ZHANG Y.J., FEI Y., STANEV E.V., GRASHORN S. (2016). Seamless cross-scale modeling with SCHISM. Ocean Modelling, Vol. 102, pp 64-81. https://doi.org/10.1016/j.ocemod.2016.05.002 
Thème 1 - Hydrodynamique côtière 